\title{
An Ultrasound-Guided Robotic HIFU Ablation System with Respiration Induced Displacement and Time Delay Compensation
}

\author{
Chih Yu An ${ }^{1}$. Yung Lun Hsu ${ }^{2}$. Ching Shiow Tseng ${ }^{1}$
}

Received: 17 September 2018 / Accepted: 28 December 2018 / Published online: 19 February 2019

(c) The Author(s) 2019

\begin{abstract}
Introduction In recent years, high-intensity focused ultrasound (HIFU) has commonly been applied in non-invasive tumor therapy, such as to treat uterine fibroids or prostate tumors. However, respiration may cause tumor displacement, such as liver tumor displacement, which may lead to errors in localization or inadequate thermal effects on the tumor. Therefore, a compensating mechanism for target localization is important.

Methods This paper introduces an ultrasound imaging-assisted robotic HIFU ablation system with a displacement compensatory mechanism. According to the correlation between the measured displacements of the heaving chest and the target tumor, a respiration simulation device was designed, which used a tumor phantom and a cam-driving mechanism to simulate displacements of the tumor and of the heaving chest. Then, a polynomial function of the tumor position relative to the position of the heaving chest was generated. After the coordinate frames of the robotic arm, optical tracker and tumor phantom had been registered, the robotic arm was able to guide the HIFU probe to track and ablate the target tumor automatically and synchronously by inputting the displacement values of the heaving chest.

Results The average positioning error in the single-point tracking experiment was $1.72 \pm 1.26 \mathrm{~mm}$, while the ablation temperature was stabilized at $80^{\circ} \mathrm{C}$. Furthermore, the average positioning error in the cross-section ablation experiment was $3.04 \pm 1.24 \mathrm{~mm}$.
\end{abstract}

Keywords High-intensity focused ultrasound $\cdot$ Robot positioning $\cdot$ Respiration $\cdot$ Ultrasound

\section{Introduction}

Non-invasive high-intensity focused ultrasound (HIFU) thermal treatment has demonstrated great potential for tumor treatment. The physical principle of this interventional approach is to apply focused ultrasound waves to the tumor tissue, such that heating of the tissue causes its necrosis [1]. The concept of HIFU was first proposed by Lynn et al. in 1942 [2], though it was not until 1960 that Fry et al. used it to treat various neurological disorders. Ultrasound was demonstrated to be able to destroy tumor cells in a region without affecting the nearby area [3]. After years of research, according to literature reviews, it is apparent that HIFU

Ching Shiow Tseng

cstseng@cc.ncu.edu.tw

1 Department of Mechanical Engineering, National Central University, Taoyuan County 32001, Taiwan, ROC

2 Graduate Institute of Biomedical Engineering, National Central University, Taoyuan County 32001, Taiwan, ROC therapy is clinically practiced to treat various cancers, such as prostate tumors [4-6], uterine fibromas [7, 8], and liver tumors [9-11].

HIFU ablation systems require a medical imaging capability and a positioning device for tumor positioning. Current HIFU ablation systems primarily use magnetic resonance imaging (MRI) or ultrasound imaging (USI) to detect the target tumor. For example, Kim et al. [12] used MRI to support a HIFU ablation system to treat 33 uterine fibroid patients. The targeting accuracy values (displacement in absolute values) in the LR, CC, and AP directions were $0.9 \pm 0.7 \mathrm{~mm}, 1.2 \pm 0.9 \mathrm{~mm}$, and $2.8 \pm 2.2 \mathrm{~mm}$, respectively. Holbrook et al. [13] focused on the distance error problem in tumor positioning due to respiration, and employed MRI to guide the HIFU focal point and track the moving phantom. Sakuma et al. [14] combined a HIFU system with an USI system, integrating an ultrasound probe with a robotic arm, using which the rotatable probe was able to obtain a 3D image of the tumor phantom. The average positional error was $3 \mathrm{~mm}$. 
However, respiration causes displacement of abdominal organs due to pressure from the diaphragm, which further affects the accuracy of tumor positioning for treatment. In fact, displacement is mostly seen in the superior-inferior direction [15]. An evaluation by Davies et al. [16] pointed out that during normal respiration, the average displacement of the liver and diaphragm in the superior-inferior direction was $10 \pm 8 \mathrm{~mm}$ and $12 \pm 7 \mathrm{~mm}$, respectively, and under deep respiration, the average displacement could be as great as $37 \pm 8 \mathrm{~mm}$ and $43 \pm 10 \mathrm{~mm}$, respectively. Song et al. [17] calculated the average displacement of the liver to be $12.8 \pm 4.6 \mathrm{~mm}$ during a respiratory cycle. Therefore, it is essential to develop a target-tracking approach to improve the positioning accuracy for HIFU ablation treatment of liver tumors during respiration.

As detection of organ displacement relies on medical imaging, and is currently limited by the acquisition time delay during HIFU treatment, it is not easy to obtain continuous data on the displacement of organs, and thus, realtime tracking is difficult. Siebenthal pointed out that an internal or external marker could be a way by which to trace the displacement of an organ during respiration [15]. An internal marker might use X-ray imaging to calculate the displacement of the diaphragm and a pulmonary tumor [18, 19]. An external marker could, for example, measure the variation in the air flow rate of the lung and its temperature [20]; assess the status of the heaving chest or abdomen using laser measurement [21,22]; or measure the change in abdominal pressure as a signal to track pulmonary tumors and abdominal lymph nodes [23]. Thus, if using an external respiratory signal to acquire the displacement of a tumor, it is then possible to compensate for such a change and continue to focus the focal point of HIFU on the target tumor, allowing the patient to breathe normally even during HIFU therapy. Seppenwoolde [24] established a model of respiratory signal and tumor displacement. In 8 patients each with a pulmonary tumor, 4 metal markers were implanted, and under normal free respiration, the position of the tumor and the state of the heaving abdomen were recorded. Then, using a polynomial function (which was obtained from those data), the displacement in the tumor position under respiration was simulated to obtain the closest trace of movement, enabling the robotic arm to continue tracking based on the model. Seo et al. [25], on the other hand, used two ultrasound probes to scan a moving kidney phantom. A coagulated lesion in the phantom was first burned and an image scanned; then, by image processing, the displacement of the lesion was calculated. In the experiment employing a robotic arm and a HIFU probe, the average positioning error of the coagulated lesion was $2 \mathrm{~mm}$.

The size of the focal point of HIFU is generally similar to that of a rice grain, which is typically smaller than the target tumor. Complete ablation of the target tumor requires a long time, and thus, the positioning of the HIFU focal point must be synchronized with the target point of the target tumor, which changes position due to respiration. The use of a robotic arm can improve the positioning accuracy and stability of the HIFU focal point. This research employed an experimental ultrasound imaging-guided robotic HIFU ablation system created for the conduction of a previous experiment [26] to further develop a synchronous method for tracking the displacement of a tumor during respiration and evaluated the accuracy of the method.

\section{Materials and Methods}

\subsection{System Structure}

Figure 1 illustrates the structure of the ultrasound imagingguided robotic HIFU ablation system. This system was based on a previously-designed experimental ultrasound imagingguided robotic HIFU ablation system [26] and combined with a respiratory simulation device in order to simulate the displacement of a tumor during the respiratory cycle. This system integrated an optical tracker (Northern Digital Inc., Polaris Spectra), dynamic reference frames (DRF, a tool with three IR reflective marker spheres), an ultrasound imaging system (Hitachi-Aloka Medical, ProSound Alpha 6), a HIFU ablation system (Sonic, H-106 probe with Instek, GFG-8255 signal generator and AR, 150A100B power amplifier), a robotic arm (YAMAHA, YK400XG), an experimental water tank, a notebook (Dell, M4500), and the novel respiratory simulation device.

An optical tracker and DRF were applied in this study for positioning purposes. The ultrasound probe scans the tumor phantom to obtain the position information of the tumor. The movement of the ultrasound probe is controlled by the motor-driven linear slide and detected by the optical tracker through the DRF, which is a reference coordinate frame tracked by the optical tracker. Through coordination transformation, the position of the tumor phantom relative to the ultrasound image frame can be transferred to and represented by the robot frame [26]. The robotic arm is thus able to bring the focal point of the HIFU transducer to aim at the tumor phantom. The signal generator and power amplifier are used to enable the HIFU transducer to generate highintensity sound power for thermal therapy.

The upper right image in Fig. 1 shows the set-up of the respiration simulation device. The heaving chest was simulated by a cam, and a phantom that represented the liver tumor was installed at the end of the linkage, which was designed according to the displacement as described in [15]. The shape of the cam was designed in accordance with the actual up-and-down changes of the chest of a real person during respiration. A full-circle rotation of the cam 


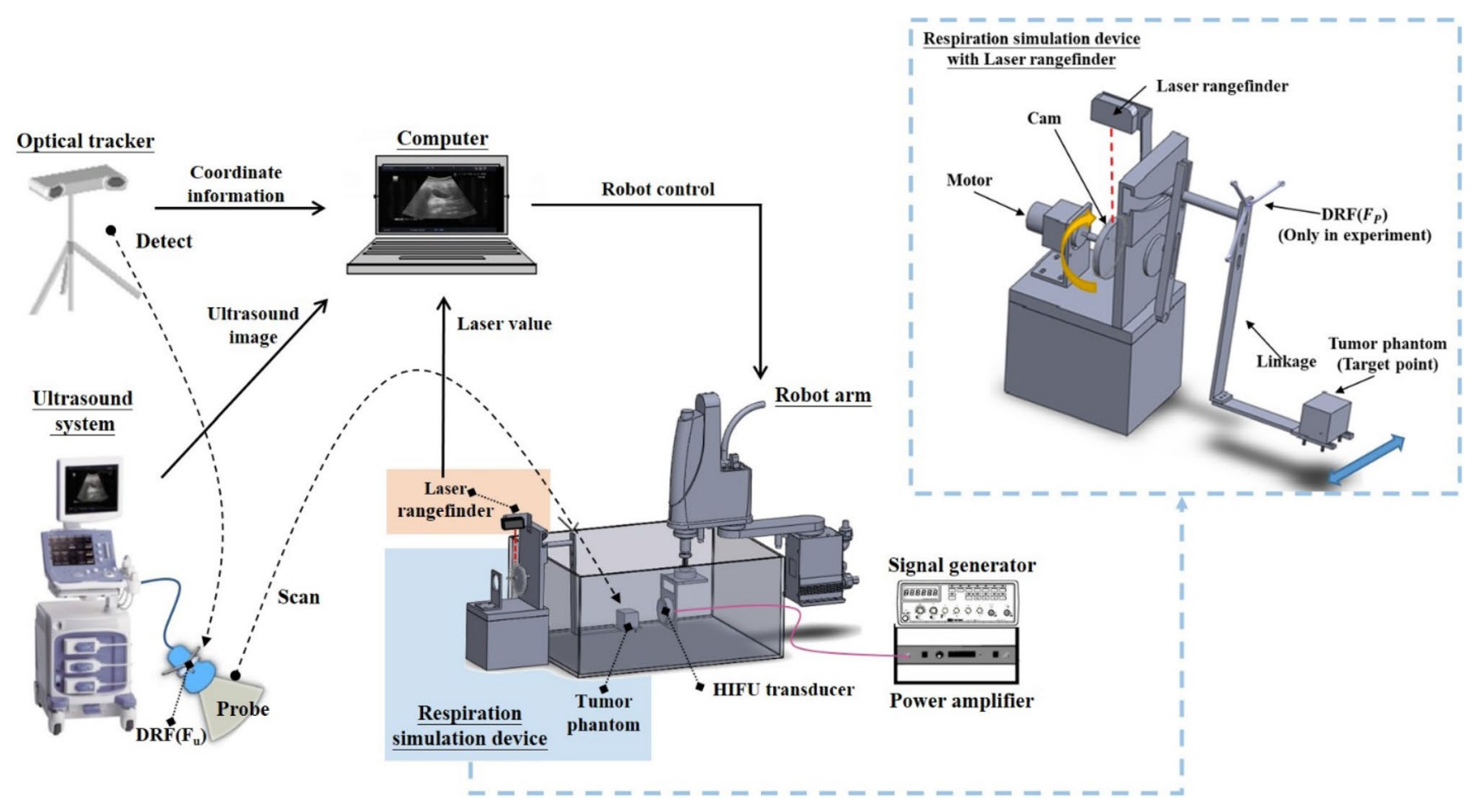

Fig. 1 System hardware architecture and respiration simulation device

driven by a motor indicated one respiratory cycle, which also generated forward-backward movement of the tumor phantom. The maximum displacement of the tumor phantom was $46.8 \mathrm{~mm}$. The change in height of the cam was measured using a laser rangefinder (Keyence, IL-100), the value of which was transmitted to the computer in order to calculate the position of the tumor phantom.

The tumor phantom was developed based on a mixed gel of acrylamide and proteins (Tung et al. [27]). According to the protein characteristics, the progress of the HIFU ablation was monitored. In addition, Takegami [28] discovered a decremental relationship between the protein volume ratio and sound speed transmission. In order to better simulate human soft tissue, the volume ratio of protein was increased to $40 \%$ from $30 \%$ to obtain an attenuation coefficient of $2.507(\mathrm{~Np} / \mathrm{m})$.

\subsection{System Execution Procedure}

A flowchart of the execution procedure of the ultrasound imaging-guided robotic HIFU ablation system is shown in Fig. 2. The procedure can be separated into three main sequential sections: acquisition of tumor images and heaving chest data, and 3D reconstruction of the tumor; establishment of the correlation between the heaving chest and tumor position; and development of a compensatory method of the robotic arm for the tracking delay to facilitate HIFU ablation. Details of the system execution procedure are given in the following sections.

\subsubsection{Tumor Imaging, Heaving Chest Positional Data Acquisition and 3D Reconstruction}

The position of the tumor changes and the chest heaves continuously during respiration. Therefore, in this study, six different heaving chest states in a respiratory cycle were chosen for position information recording and scanning of the tumor using an ultrasound probe. The six different states were as follows: maximal exhalation; maximal inhalation; 2 during the exhalation process; and 2 during the inhalation process. Meanwhile, positional information for the heaving chest was obtained by laser rangefinder measurement. Subsequently, the ultrasound images of the tumor were subjected to image processing. Along with its position information, we employed the marching cube method to reconstruct the tumor as a 3D model (Fig. 3). Details of the image processing and 3D reconstruction procedures are presented below.

In the original tumor ultrasound images detected by the ultrasound probe, it was not easy to detect the edge position information directly due to noise. In order to obtain the edge position information for 3D reconstruction, image denoising was the first priority in image processing. First, we determined the region of interest (ROI) in each ultrasound 
Fig. 2 System execution flowchart

Fig. 3 Image processing and 3D reconstruction of the tumor
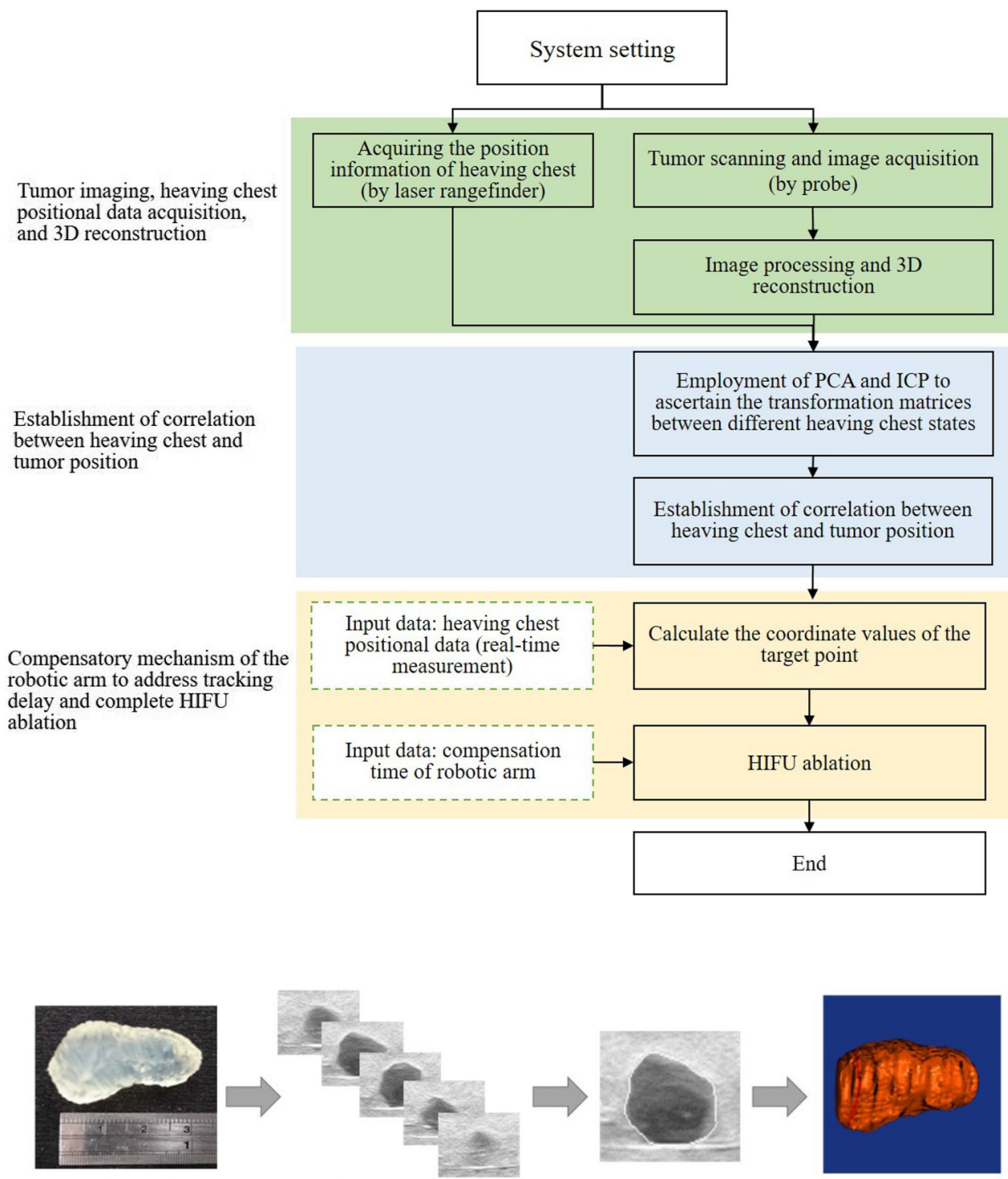

Tumor (Tumor phantom)
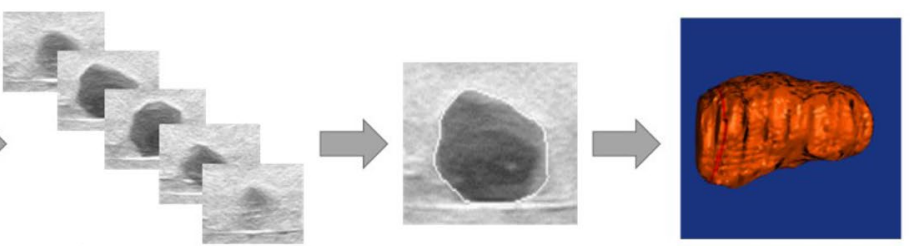

Ultrasound scanning

3D model Image processing reconstruction

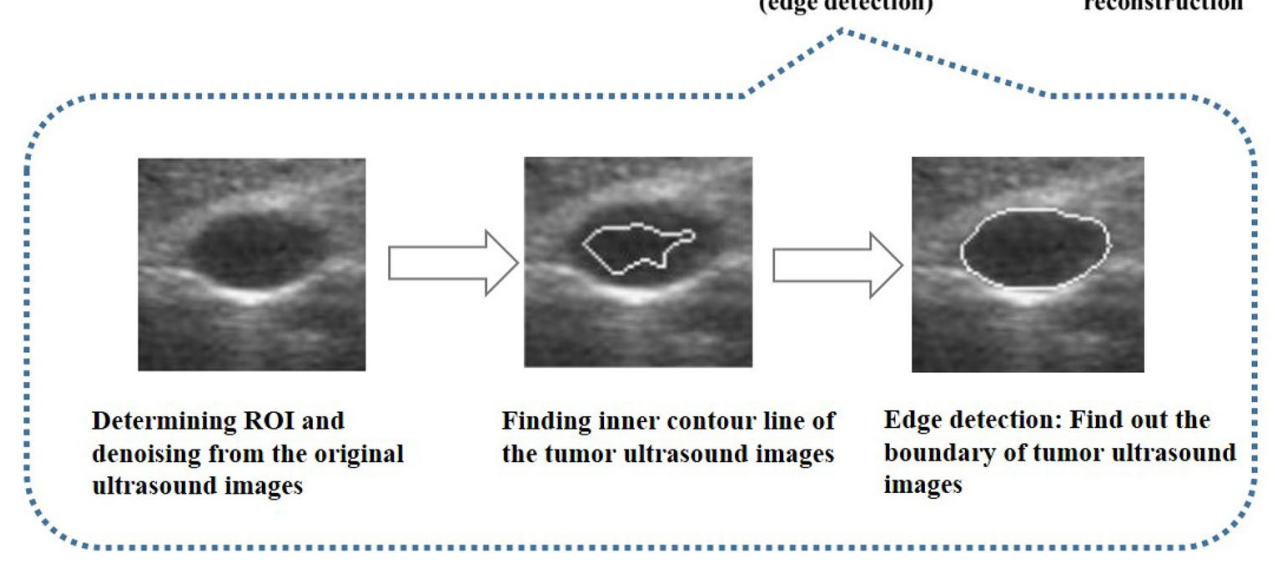

image. Then, the erosion-dilation method was applied to the ROI of the original tumor ultrasound images for denoising. Subsequently, for boundary-finding purposes, the inner contour line of the tumor image had to be identified first. Otsu's method was used in this study to ascertain threshold value, using which the tumor ultrasound images could be thresholded, following by Canny edge detection to ascertain the inner contour line of the tumor ultrasound images. Next, the 
boundary of the tumor ultrasound image was identified using marker-controlled watershed segmentation, under which the inner contour line of the tumor ultrasound image was taken as the inner marker and the boundary of the ROI was taken as the outer marker. Finally, the position information of the tumor had already been obtained by the optical tracker during scanning of the tumor. Therefore, the boundary information of the tumor ultrasound images (after image processing) could also be obtained by calculation. Along with this boundary position information, we employed the marching cube method to reconstruct the tumor as a 3D model.

OpenCV was selected as the tool for image processing in this study. The video capture card in this system was UPG306 , the ultrasound image resolution was $640 * 480$, and the capture speed was $30 \mathrm{fps}$. In this study, approximately 300 ultrasound images were captured during each scan, and the image processing duration was approximately $15 \mathrm{~s} /$ per scan (of 300 ultrasound images). The resolution of the ROI in each tumor ultrasound image was approximately $90 * 90$. The Visualization Toolkit (VTK) was selected as the tool for 3D reconstruction in this study, and the $3 \mathrm{D}$ reconstruction duration for one tumor in one position state was approximately 10 s (using Win 7 OS; CPU i72600; resolution of $640 * 480$ ).

\subsubsection{Establishment of the Correlation Between the Heaving Chest and Tumor Position}

In order to obtain the tumor position from the positional information of the heaving chest, it was necessary to ascertain the correlative polynomial function between the heaving chest and the tumor position. First, the transformation matrices among the 3D tumor model frames under different heaving chest states were ascertained using the Iterative Closest Points (ICP) algorithm. By inputting the Source $\mathrm{S}=\left\{P_{s_{i}}, i=0,1,2, \ldots, k\right\}$ and the Target $\mathrm{T}=\left\{P_{t_{i}}, i=0,1,2, \ldots, u\right\}$, the transformation relationship between $\mathrm{S}$ and $\mathrm{T}$ was obtained. Equation 1 shows that the coordinates between the points in the S-group and T-group were correlated by the transformation matrix $T_{s}^{t}$ between $F_{t}$ (frames of the T-group) and $F_{s}$ (frames of the S-group).

$P_{t}=T_{s}^{t} P_{s}$

Following establishment of the transformation matrix, any target ablation point $P_{s}$ under frame $F_{s}$ could be transformed by applying the transformation matrix $T_{s}^{t}$ to obtain $P_{t}$ (which is the ablation point under frame $F_{t}$ ). S and T represent different heaving chest states. Thus, finding the transformation matrix between two heaving chest states renders it a simple task to calculate the coordinate information of the target ablation point in the subsequent heaving chest state. The transformation relationship between $\mathrm{S}$ and $\mathrm{T}$ is shown in Fig. 4a.

Another key variable used to generate the heaving-chestand-tumor-position polynomial function was the position value of the heaving chest $L_{j}$, where " $j$ " represented the heaving chest state (e.g., $j=0$, maximal exhalation). The 3rd-order polynomial function was generated from the corresponding positions of the target point $P_{t j}$ and the heaving chest $L_{j}$ in each of the $\mathrm{X}, \mathrm{Y}$, and $\mathrm{Z}$ directions. Then, the heaving chest and tumor position correlation was established in each exhalation and inhalation state (Fig. 4b).

\subsubsection{Compensatory Mechanism of the Robotic Arm for Tracking Delay and HIFU Ablation}

Ideally, the robotic arm would be able to track the tumor accurately according to the heaving-chest-and-tumor-position polynomial function calculation results. However, the system operation procedure, information transmission, computer calculation and robotic arm movement caused tracking delay of the robotic arm (Fig. 5), which affected the accuracy

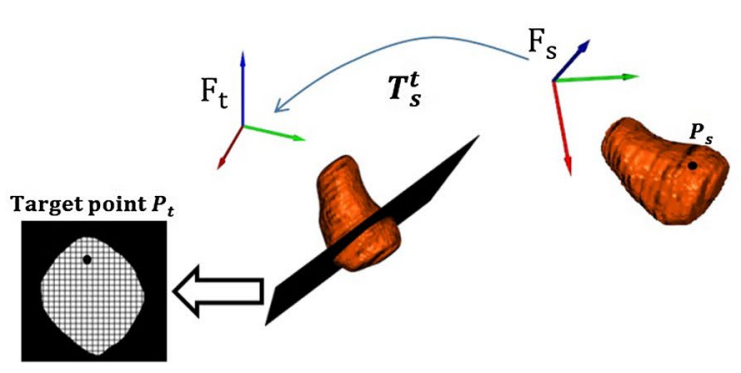

(a)

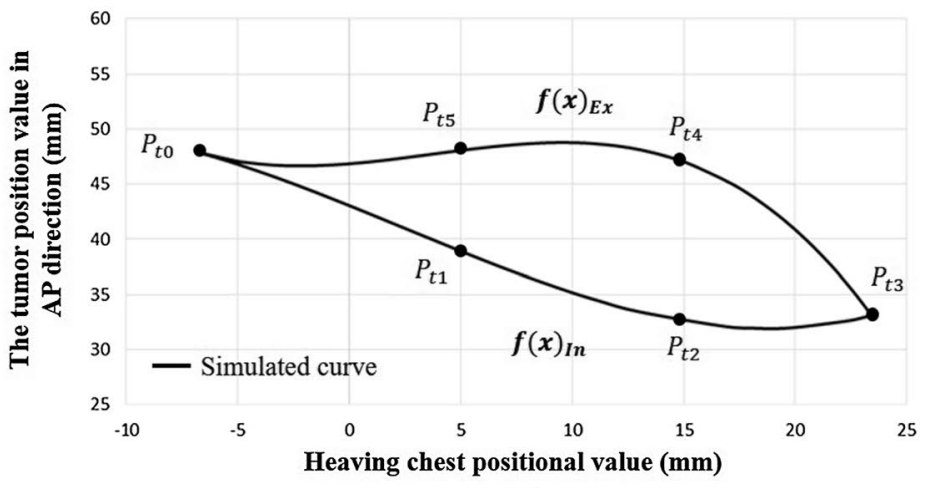

(b)

Fig. 4 a Transformation relationship of the scheduled target point of the frame system. b Relationship between the position value of the heaving chest and tumor position 


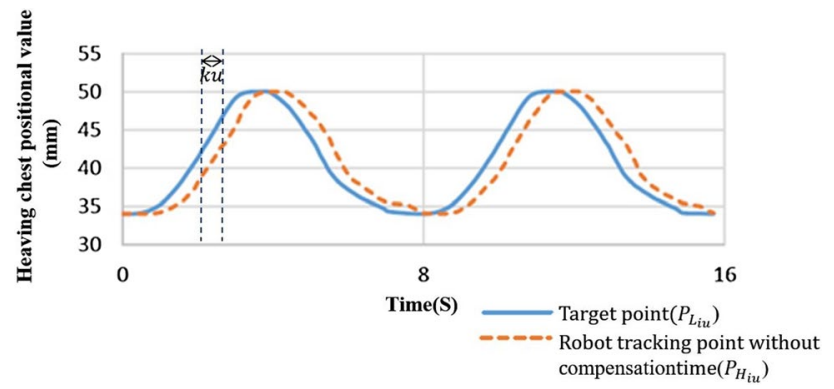

Fig. 5 Illustration of the positional delay in tracking of the target point by the robotic arm

of ablation. Therefore, the establishment of a compensatory mechanism was necessary in this case.

The method employed to calculate the delay was to record the position value of the HIFU focal point $\left\{P_{H_{i u}}\right\}$ without a compensatory mechanism by setting a DRF at the end of the robotic arm ( $i$ : the number of the points; $u$ : the sampling interval, with $0.1 \mathrm{~s}$ as the unit time). The results were compared with the tumor target position $\left\{P_{L_{i u}}\right\}$, which was calculated using the heaving-chest-and-tumor-position polynomial function along with the position value of the chest (Fig. 4b). As shown in Fig. 5, there existed a time delay between HIFU focal point position $P_{H_{i u}}$ and target point $P_{L_{i u}}$. In order to ascertain the compensation time, $k u$ was defined as the compensation time at the beginning ( $k$ : an unknown integer; $k=0,1,2, \ldots$ ). An iterative search for the $k u$ value was executed until the minimal summation error between $P_{H_{i u}}$ and $P_{L_{i u}}$ was found (Eq. 2). At this time, $k u$ was taken to be the compensation time required for the robot to track the movement of target point.

$\min \sum_{i}^{N}\left\|P_{H_{(i+k) u}}-P_{L_{i u}}\right\| \mathrm{N}$ : number of target points

\section{Results of Experiments}

This research used a respiration simulation device to simulate the movement of the heaving chest, as well as the positional displacement of a liver tumor (using a tumor phantom). A DRF was set on the linkage of the respiration simulation device, as shown in the upper right image in Fig. 1, in order to track and detect the coordinates of the tumor phantom. Before the final experiment, the compensation time for the robotic arm had to be obtained in order to minimize the tracking error. The final experiment was conducted to evaluate the synchronized tracking accuracy. The experiment was separated into two parts: single-point tracking and cross-sectional ablation. Both used a thermocouple to measure the ablation temperature, and the tracking accuracy was judged according to the coagulation of the lesion.

\subsection{Robotic Arm Tracking Compensation Experiment}

The rotational speed of the respiration simulation device was driven by a motor, and the cycle duration was set to $9 \mathrm{~s}$, which matched the normal respiratory cycle duration of an adult. During the experiment, the robotic arm controlled the HIFU focal point to track a target point (on the tumor phantom). The moving positions of the HIFU focal point and the target point were recorded simultaneously during a respiration cycle, as shown in Fig. 6a. These heaving chest positional data were then imported into the heaving-chestand-tumor-position polynomial function to calculate the compensation time in order to minimize the distance error. Figure $6 \mathrm{~b}$ shows that the focal point and the target point had the minimal distance error when the compensation time was $0.2 \mathrm{~s}$.

After the compensation time had been found, the accuracy of target point tracing during three respiratory cycles was measured. The correlation between the heaving chest state and the compensated HIFU tracking error was as shown in Fig. 6c. The average tracking error after time compensation was $0.65 \pm 0.31 \mathrm{~mm}$.

\subsection{Synchronous Tracking and Ablation Experiment}

\subsubsection{Single-Point Tracking and Ablation Experiment}

As shown in Fig. 7a, a thermocouple was inserted into the tumor phantom, the top of which was the target point in this experiment. A needle-type probe of the optical tracker measured the tip of the thermocouple (the target point) in order to obtain the coordinates of the target point, which were then used as a control group and compared with those calculated using the heaving-chest-and-tumor-position polynomial function to obtain the tracking error of the HIFU focal point.

In the experiment, the respiration simulation device was activated for $45 \mathrm{~s}$, and the HIFU focus point driven by the robotic arm tracked and ablated the target point simultaneously. The temperature of the target point was recorded continuously in addition to the positioning error of the HIFU focal point, and the results are shown in Fig. 7b. The overall temperature was around $80{ }^{\circ} \mathrm{C}$, which would be sufficient to kill tumor cells in a real case. The results also showed that when the positioning error increased, the temperature decreased significantly. The relationship between the HIFU focal point positioning error and the heaving chest state was investigated in a subsequent experiment, as described below. 


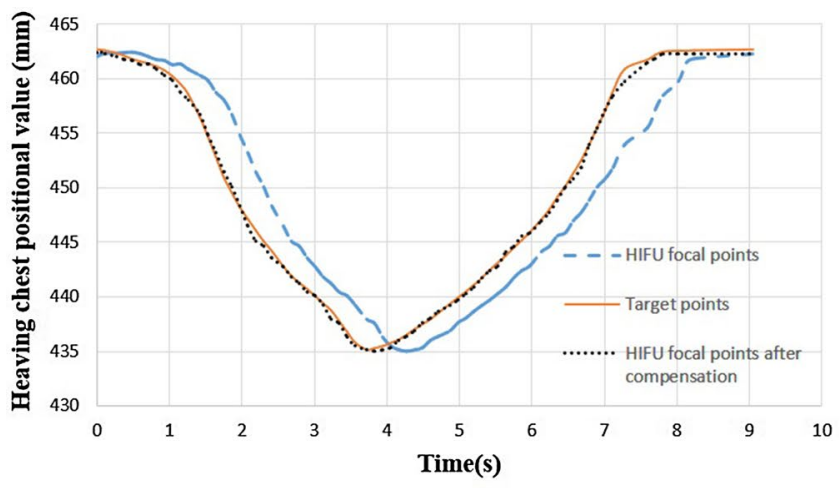

(a)

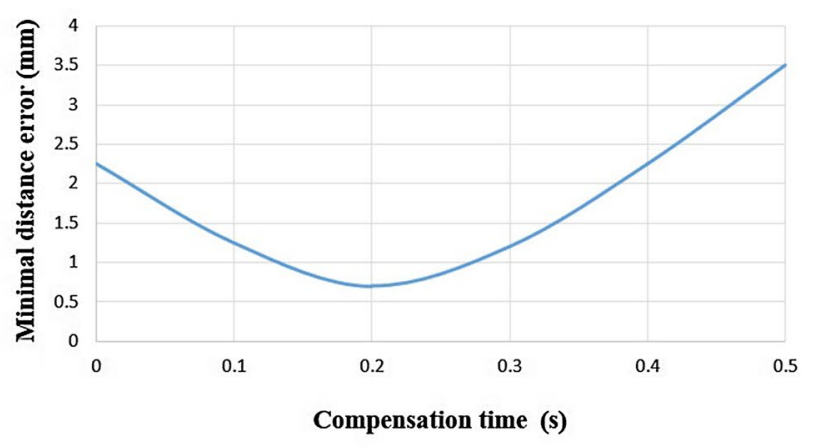

(b)

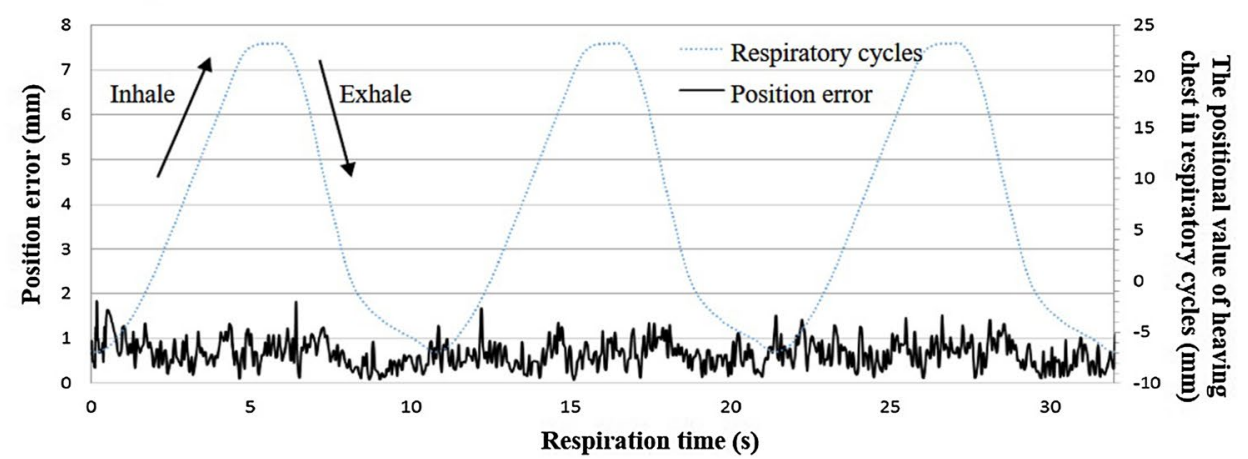

(c)

Fig. 6 a Correlation between the target points and focus points before and after compensation. b Correlation between the compensation time and the minimal average distance error. $\mathbf{c}$ HIFU tracking error after time compensation

A duration of three respiratory cycles was applied as the basis of the experiment conducted to investigate the relationship between the HIFU positioning error and the heaving chest state. The average positioning error over three respiratory cycles was $1.72 \pm 1.26 \mathrm{~mm}$. Figure $7 \mathrm{c}$ shows every positioning error and the position values of the heaving chest during the three respiratory cycles. Repeatability in positioning error was observed. In addition, significantly increasing positioning errors occurred during the inhalation and exhalation transition periods (blue dotted frames). This can be explained by the difference between the simulated trajectory and the actual trajectory of the target point, as detailed below.

\subsubsection{Cross-Section Ablation Experiment}

In order to more closely represent the real situation, Fig. 8a shows an oval-shaped tumor phantom $(4 \mathrm{~cm}$ in length, $2 \mathrm{~cm}$ in width) that was wrapped in graphite agar and placed at the end of the linkage. The experimental process was the same as that used for the previous experiment. An optical tracker was also employed to track the coordinates of the tumor phantom, and the results were compared with the coordinates calculated using the heaving-chest-and-tumorposition polynomial function to obtain the positioning error of the HIFU focal point. In addition, a cross-section of the $3 \mathrm{D}$ tumor model was chosen as the target plane. Figure $8 \mathrm{~b}$ shows the 25 points on the plane chosen to be ablated; the distance between each point was $2 \mathrm{~mm}$, and the ablation route was of an "S" shape.

In the experiment, the respiration simulation device ran for 7 respiration cycles in order to ablate 25 target points. The average positioning error after importing the compensation time was $3.04 \pm 1.24 \mathrm{~mm}$. The results of the average positioning error and the position value of the heaving chest during the different heaving chest states are presented in Fig. 8c. It was observed that there was regularity in the positioning error, and larger positioning errors occurred during the inhalation and exhalation transition periods.

Figure 8d shows the planned route for ablation on the cross-section and the cross-section of the tumor phantom after ablation. The white coagulated lesion in the area of the 


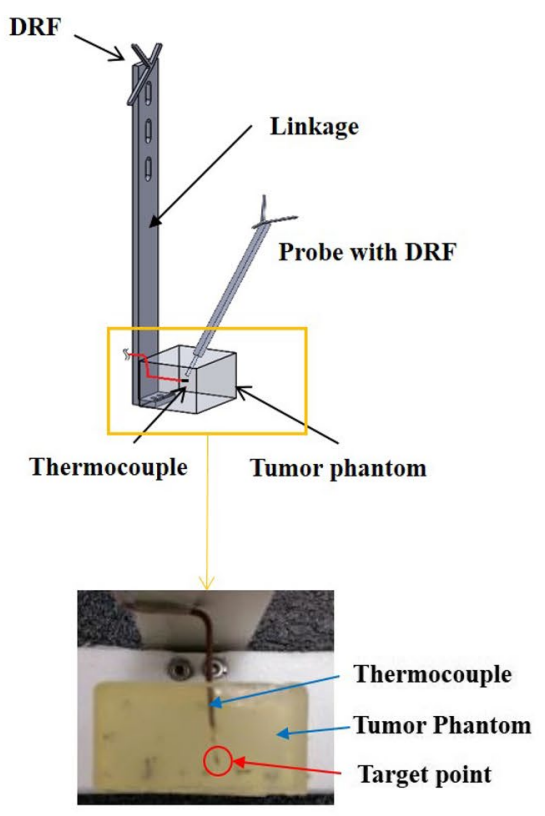

(a)

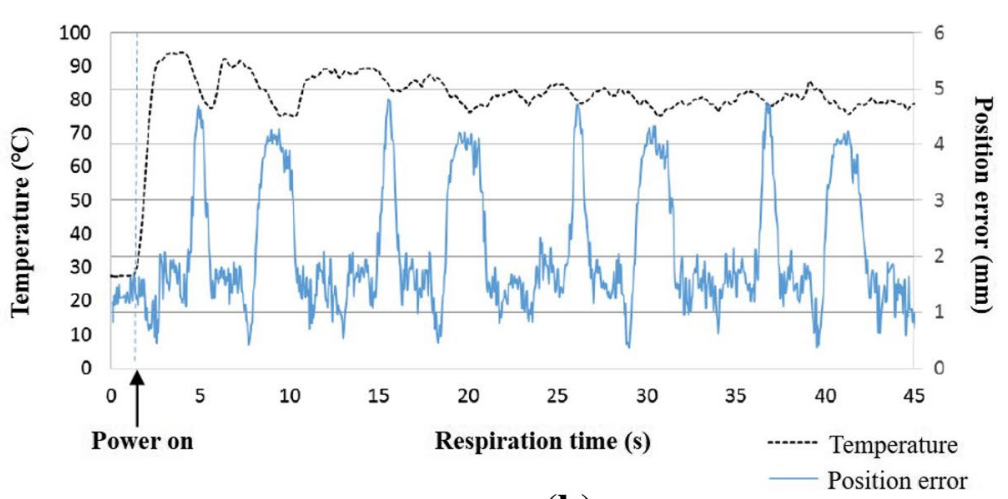

(b)

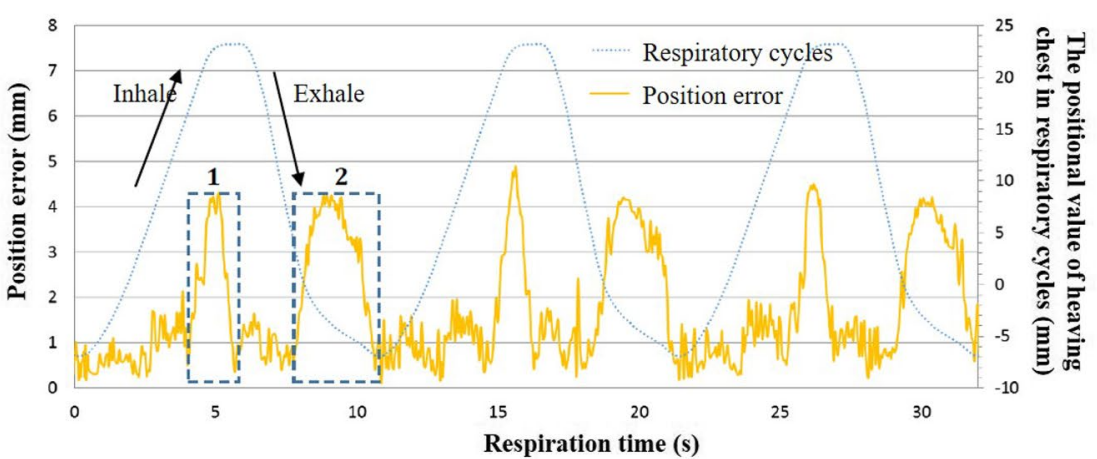

(c)

Fig. 7 a Arrangement of the tumor phantom and thermocouple. b Variations of temperature and positioning error during ablation. $\mathbf{c}$ Relationship between positioning error and heaving chest state

planning route was obvious (owing to protein denaturation when the temperature was above $60{ }^{\circ} \mathrm{C}$ ).

\section{Discussion and Conclusion}

To summarize the experimental results, the robot compensation time was $0.2 \mathrm{~s}$, and the average positioning error in the single-point ablation experiment was $1.72 \pm 1.26 \mathrm{~mm}$, allowing the ablation temperature at the target point to remain stabilized at $80{ }^{\circ} \mathrm{C}$, denaturing the proteins in the tumor phantom. Moreover, the results of the 3D crosssection ablation experiment showed an average positioning error of $3.04 \pm 1.24 \mathrm{~mm}$, which was larger than that observed in the single-point ablation experiment. In addition, in both the single-point ablation experiment and the cross-section ablation experiment, larger positioning errors occurred at the moment of change from inhalation to exhalation. It was speculated that the number of sampling points was too few to enable establishment of the heaving-chest-and-tumor-position polynomial function, resulting in positioning error between the simulated trajectory and the actual trajectory. However, according to the ablation results of the tumor phantom, this system achieved the expected outcome, the target ablation region being filled with the white coagulated lesion.

In this research, an ultrasound-guided robotic HIFU ablation system with respiration displacement and time delay compensation functions was developed. The main characteristic of this system to note is that the robotic arm guided the HIFU focal point to track and ablate the target point automatically and synchronously by importing the position values of the heaving chest and the compensation times. In future study, the number of tumor sampling positions will be increased in order to better approximate the actual tumor trajectory by the simulation trajectory, and real animal tissue will be used for further testing. In addition, different HIFU powers, tumor tissue categories, and thicknesses will be included as factors in order to evaluate the variation in positioning accuracy of the HIFU focal point. 


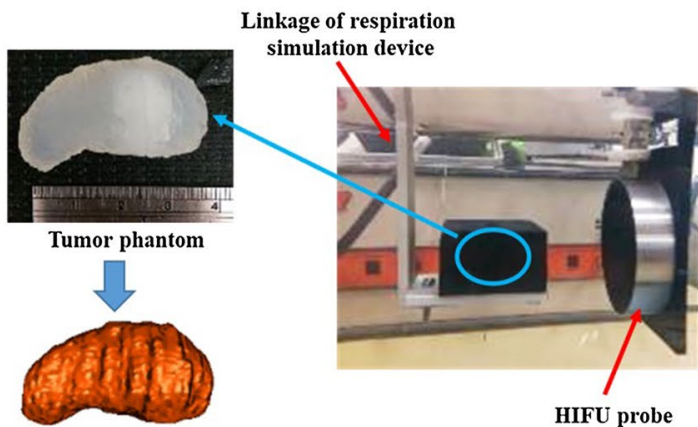

(a)

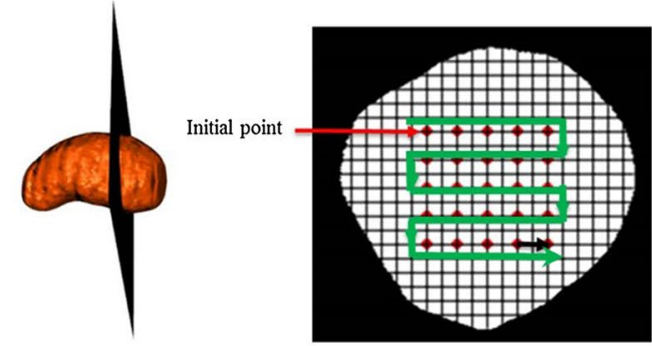

(b)

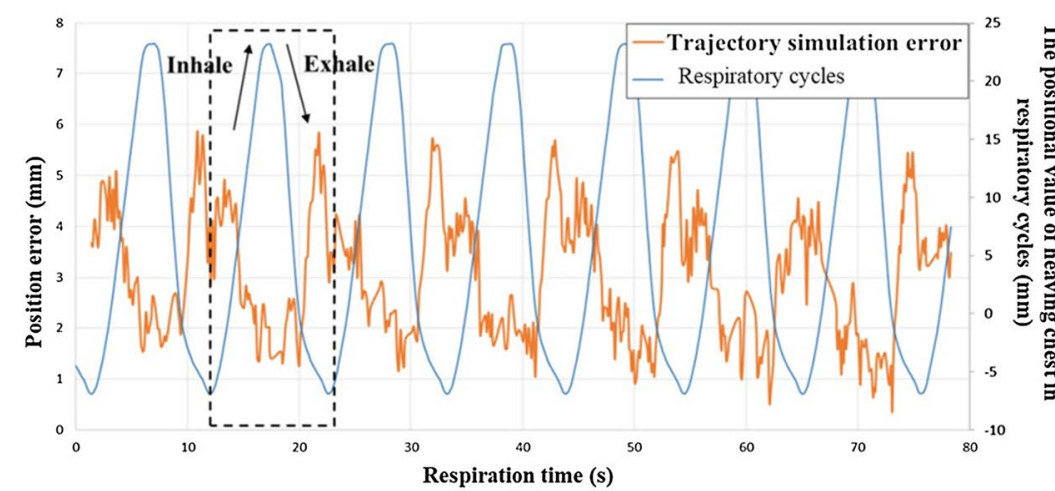

(c)
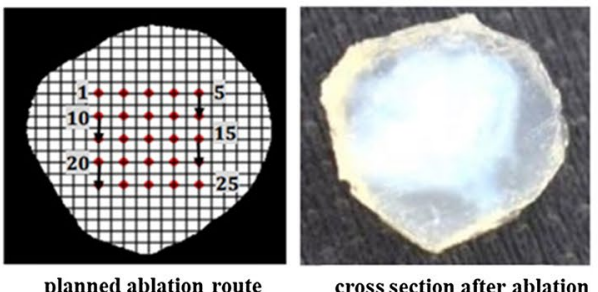

(d)

Fig. 8 a Tumor phantom and $\mathbf{b}$ ablation plane for the cross-sectional ablation experiment. $\mathbf{c}$ Positioning error during synchronous tracking. $\mathbf{d}$ Comparison of ablation area

Acknowledgements The authors would like to thank the Ministry of Science and Technology, Taiwan, for its financial support under the contract NSC 101-2221-E-008-020-MY3 and MOST 105-2221-E-008-041-MY2.

\section{Compliance with Ethical Standards}

Conflict of interest The authors declare that there are no conflicts of interest regarding the publication of this paper.

OpenAccess This article is distributed under the terms of the Creative Commons Attribution 4.0 International License (http://creativeco mmons.org/licenses/by/4.0/), which permits unrestricted use, distribution, and reproduction in any medium, provided you give appropriate credit to the original author(s) and the source, provide a link to the Creative Commons license, and indicate if changes were made.

\section{References}

1. Seo, J., Koizumi, N., Yoshinaka, K., Sugita, N., Nomiya, A., Homma, Y., et al. (2010). Three-dimensional computer-controlled acoustic pressure scanning and quantification of focused ultrasound. IEEE Transactions on Ultrasonics, Ferroelectrics, and Frequency Control, 57(4), 883-891.
2. Lynn, J. G., Zwemer, R. L., Chick, A. J., \& Miller, A. E. (1942). A new method for the generation and use of focused ultrasound in experimental biology. Journal of General Physiology, 26(2), 179-193.

3. Fry, W. J., \& Fry, F. J. (1960). Fundamental neurological research and human neurosurgery using intense ultrasound", Medical Electronics. IRE Transactions on Medical Electronics., 7(3), 166-181.

4. Filipek, M., Czarniecki, S., \& Lewicki, A. (2014). The assessment of PSA concentration dynamics in prostate cancer patients treated with radical-HIFU in own material. European Urology Supplements, 13(6), e1212.

5. Czarniecki, S., Lewicki, A., \& Filipek, M. (2014). Prostate biopsy in patients following High Intensity Focused Ultrasound (HIFU) therapy for prostate cancer in own material. European Urology Supplements, 13(6), 1217.

6. Veereman, G., Jonckheer, P., Desomer, A., Brabandt, H. V., D'Hont, C., Velthoven, R. V., et al. (2015). Systematic review of the efficacy and safety of high-intensity focussed ultrasound for localised prostate cancer. European Urology Focus, 1(2), $158-170$.

7. Zhang, X., Li, K., Xie, B., He, M., He, J., \& Zhang, L. (2014). Effective ablation therapy of adenomyosis with ultrasoundguided high-intensity focused ultrasound. International Journal of Gynecology \& Obstetrics, 124(3), 207-211.

8. Shui, L., Mao, S., Wu, Q., Huang, G., Wang, J., Zhang, R., et al. (2015). High-intensity focused ultrasound (HIFU) for 
adenomyosis: Two-year follow-up results. Ulreasonics Sonochemistry, 27, 677-681.

9. Illing, R. O., Kennedy, J. E., Wu, F., Ter Haar, G. R., Protheroe, A. S., Friend, P. J., et al. (2005). The safety and feasibility of extracorporeal high-intensity focused ultrasound (HIFU) for the treatment of liver and kidney tumours in a Western population. British Journal of Cancer, 93(8), 890-895.

10. Zhang, L., Zhu, H., Jin, C., Zhou, K., Li, K., Su, H., et al. (2009). High-intensity focused ultrasound (HIFU): effective and safe therapy for hepatocellular carcinoma adjacent to major hepatic veins. European Radiology, 19(2), 437-445.

11. Dupré, A., Melodelima, D., Chen, Y., Pérol, D., Kocot, A., Vincenot, J., et al. (2016). Intra-operative ablation of colorectal liver metastases (CLM) with high intensity focused ultrasound (HIFU): Preliminary results of a prospective ablate-and-resect study. $H P B$, 18(S1), e130.

12. Kim, Y., Trillaud, H., Rhim, H., Lim, H. K., Mali, W., Voogt, M., et al. (2012). MR thermometry analysis of sonication accuracy and safety margin of volumetric MR imaging-guided high-intensity focused ultrasound ablation of symptomatic uterine fibroids. Radiology, 265(2), 627-637.

13. Holbrook, A. B., Ghanouni, P., Santos, J. M., Dumoulin, C., Medan, Y., \& Pauly, K. B. (2014). Respiration based steering for high intensity focused ultrasound liver ablation. Magnetic Resonance in Medicine, 71(2), 797-806.

14. Sakuma, I., Takai, Y., Kobayashi, E., Inada, H., Fujimoto, K., \& Asano, T. (2002). Navigation of high intensity focused ultrasound applicator with an integrated three-dimensional ultrasound imaging system. MICCAI, 2489, 133-139.

15. Siebenthal, M. V. (2008). Analysis and modelling of respiratory liver motion using 4DMRI. Konstanz: Hartung-Gorre Verlag.

16. Davies, S. C., Hill, A. L., Holmes, R. B., Halliwell, M., et al. (1994). Ultrasound quantitation of respiratory organ motion in the upper abdomen. The British Journal of Radiology, 67, 1096-1102.

17. Song, R., Tipirneni, A., Johnson, P., et al. (2011). Evaluation of respiratory liver and kidney movements for MRI navigator gating. Journal of Magnetic Resonance Imaging, 33, 143-148.
18. Cui, Y., et al. (2007). Multiple template-based fluoroscopic tracking of lung tumor mass without implanted fiducial markers. Physics in Medicine \& Biology, 52(20), 6229-6242.

19. Cerviño, L. I., et al. (2009). The diaphragm as an anatomic surrogate for lung tumor motion. Physics in Medicine \& Biology, 54(11), 3529-3542.

20. Hoisak, J. D. P., et al. (2004). Correlation of lung tumor motion with external surrogate indicators of respiration. International Journal of Radiation Oncology Biology Physics, 60(4), 1298-1306.

21. Berbeco, R. I., et al. (2006). Residual motion of lung tumors in end-of-inhale respiratory gated radiotherapy based on external surrogates. Medical Physics, 33(11), 4149-4156.

22. Ionascu, D., et al. (2007). Internal-external correlation investigations of respiratory induced motion of lung tumors. Medical Physics, 34(10), 3893-3903.

23. Otani, Y., et al. (2010). A comparison of the respiratory signals acquired by different respiratory monitoring systems used in respiratory gated radiotherapy. Medical Physics, 37(12), 6178-6186.

24. Seppenwoolde, Y., et al. (2007). Accuracy of tumor motion compensation algorithm from a robotic respiratory tracking system: a simulation study. Medical Physics, 34(7), 2774-2784.

25. Seo, J., et al. (2011). Visual servoing for a US-guided therapeutic HIFU system by coagulated lesion tracking: A phantom study. The International Journal of Medical Robotics and Computer Assisted Surgery, 7(2), 237-247.

26. An, C. Y., Syu, J. H., Tseng, C. S. et al. (2017). An ultrasound imaging-guided robotic HIFU ablation experimental system and accuracy evaluations. Applied Bionics and Biomechanics, 2017.

27. Tung, Y. S., Liu, H. L., Wu, C. C., et al. (2006). Contrast-agentenhanced ultrasound thermal ablation. Ultrasound in Medicine and Biology, 32, 1103-1110.

28. Takegami, K., Kaneko, Y., Watanabe, T., et al. (2004). Polyacrylamide gel containing egg white as new model for irradiation experiments using focused ultrasound. Ultrasound in Medicine and Biology, 30, 1419-1422. 